\title{
NONLINEAR ACCRETIVE OPERATORS IN BANACH SPACES
}

\author{
BY FELIX E. BROWDER
}

Communicated December 8, 1966

Introduction. Let $X$ be a real Banach space, $X^{*}$ its conjugate space with the pairing between $w$ in $X^{*}$ and $x$ in $X$ denoted by $(x, w)$. The duality mapping $J$ of $X$ into $2^{x^{*}}$ is given by

$$
J(x)=\left\{w \mid w \in X^{*} ;\|w\|=\|x\| ;(x, w)=\|x\| \cdot\|w\|\right\},
$$

for each $x$ in $X$. For any Banach space $X$ and any element $x$ of $X$, $J(x)$ is a nonempty closed convex subset of the sphere of radius $\|x\|$ about zero in $X^{*}$. If $X^{*}$ is strictly convex, $J$ is a singlevalued mapping of $X$ into $X^{*}$ and is continuous from the strong topology of $X$ to the weak* topology of $X^{*} . J$ is continuous in the strong topologies if and only if the norm in $X$ is $C^{1}$ on the complement of the origin.

Definition. If $T$ is a (possibly) nonlinear mapping with domain $D(T)$ in $X$ and with range $R(T)$ in $X$, then $T$ is said to be accretive if for each pair $x$ and $y$ in $D(T)$,

$$
(T(x)-T(y), J(x-y)) \geqq 0,
$$

i.e. $(T(x)-T(y), w) \geqq 0$ for all $w$ in $J(x-y)$.

If $X^{*}$ is strictly convex, the nonlinear accretive operators from $X$ to $X$ coincide with the $J$-monotone operators studied in Browder [3], [4] and Browder-de Figueiredo [8]. For linear $T, T$ is accretive if and only if $(-T)$ is dissipative in the sense of Lumer-Phillips [10]. Functional equations for nonlinear accretive operators have also been considered by Vainberg [15] and related classes of operators and problems have been studied by Hartman [9], Mamedov [11], Murakami [13], and Petryshyn [14].

It is our object in the present paper to present a number of general existence theorems for solutions of nonlinear functional equations involving nonlinear accretive operators which drastically improve earlier results in this direction as given in the papers mentioned above. The detailed proofs of these general existence theorems are given in another paper [7]. In the second section of the present paper, we give under somewhat sharper hypotheses a procedure of projectional or Galerkin type for computing such solutions. The convergence proof which is given in full is of special interest because it depends in an essential way upon the fact that the existence theorem for solutions has been given an independent proof. (We should also remark that the proofs of the existence theorems of [7] are also constructive but in a more complicated fashion.) 
When $X$ is a Hilbert space, $J=I$, and the accretive operators reduce to the class of monotone operators. The special interest of the class of nonlinear accretive operators $T$ with domain and range in a Banach space $X$ is that under the hypotheses of the Theorems below, the operators $(-T)$ coincide with the infinitesimal generators of oneparameter $C_{0}$ semigroups of nonlinear contraction operators in $X$, (i.e. of nonlinear operators $\{U(t), t \geqq 0\}$ such that for all $x$ and $y$ in $X$, $\|U(t) x-U(t) y\| \leqq\|x-y\|$. We distinguish contractions or nonexpansive operators in the terminology of Browder [3] from strict contractions $V$ for which there exists a constant $k<1$ such that $\|V(x)-V(y)\|$ $\leqq k\|x-y\|,(x, y \in X))$. Nonlinear accretive operators extend this property of monotone operators in Hilbert space, rather than the characteristic properties of gradients of functionals on $X$, as for the class of monotone operators $T$ from $X$ to $X^{*}$. (For a survey of the literature on the latter class, cf. [1], [2], [5], [6].)

1. The present section is devoted to the statement of the general existence theorems, in a simple operator-theoretical form.

THEOREM 1. Let $X$ be a Banach space with a strictly convex conjugate space $X^{*}$ and with the duality mapping $J$ uniformly continuous from the unit sphere in the strong topology of $X$ to the strong topology of $X^{*}$. Let $T$ be an operator with domain and range in $X$ of the form $T=-L$ $+T_{0}$, where $L$ is a closed densely defined linear operator in $X$ which is the infinitesimal generator of a $C_{0}$ contraction semigroup, while $T_{0}$ is a continuous nonlinear accretive mapping of $X$ into $X$ which maps bounded sets into bounded sets. Suppose that the inverse image under $T$ of each bounded set is bounded.

Then $R(T)$, the range of $T$, is dense in $X$.

THEOREM 2. Suppose that in addition to the hypotheses of Theorem 1, we assume that for each bounded subset $B$ of $X$, there exists a compact mapping $C$ of $B$ into $X$ and a strictly increasing continuous function $\lambda$ from $R^{+}=\{t \mid t \geqq 0\}$ to $R^{+}$with $\lambda(0)=0$, such that

(3) $\|(T+C) x-(T+C) y\| \geqq \lambda(\|x-y\|) ; \quad(x, y \in B \cap D(T))$.

Then the range of $T$ is all of the space $X$.

ThEOREM 3. In Theorems 1 and 2, the hypothesis on the boundedness of inverse images under $T$ of bounded sets in $X$ can be replaced by the stronger hypothesis of $J$-coerciveness of $T$, namely:

$$
(T(u), J(u)) /\|u\| \rightarrow+\infty \quad(\|u\| \rightarrow+\infty, u \in D(T)) .
$$

A corollary of the preceding results is the following generalization of a theorem of Minty in Hilbert space [12]: 
THEOREM 4. Let $X$ be a Banach space with strictly convex $X^{*}$ and $J$ uniformly continuous on the unit sphere. Let $T=-L+T_{0}$, with $L$ the linear generator of a contraction semigroup, $T_{0}$ a nonlinear accretive operator which is continuous from $X$ to $X$ and maps bounded sets into bounded sets.

Then $T+k I$, for any $k>0$, maps $D(T)$ onto $X$ and has a Lipschitzian inverse mapping $X$ into $X$.

TheOREM 5. In Theorems 1, 2, 3, and 4, if the Banach space $X$ is reflexive, the continuity condition on $T_{0}$ can be replaced by demicontinuity, i.e. $T_{0}$ is continuous from the strong topology of $X$ to the weak topology of $X$.

Theorem 6. Suppose that under the hypotheses of Theorem 1, we assume that there exists a mapping $J_{1}$ of $X$ into $X^{*}$ of the form $J_{1}(x)$ $=\mu(\|x\|) J(x)$ with $\mu(r)>0$ for $r>0$ such that $J_{1}$ is continuous from the weak topology of $X$ to the weak topology of $X^{*}$. Suppose that $X$ is reflexive and $T_{0}$ merely demicontinuous.

Then the range of $T$ is all of $X$.

Theorem 6 contains as a special case for $L=0$, the result of Browder and de Figueiredo [8] obtained under the additional condition that $X$ is separable and satisfies condition $(\pi)_{1}$ of $\$ 2$ below. A very special case of Theorem 2 was established by Vainberg [15] for $L=0, T_{0}$ satisfying a Lipschitz condition on each bounded set, and $T=T_{0}$ satisfying the inequality

$$
(T(x)-T(y), J(x-y)) \geqq m(r)\|x-y\|^{2}, \quad(\|x\|,\|y\| \leqq r),
$$

with $m(t)$ a positive decreasing function on $R^{+}$such that $t m(t) \rightarrow+\infty$ as $t \rightarrow+\infty$.

The proofs of our results are based upon a generalized method of steepest descent obtaining the convergence of solutions of differential equations of the form $d u / d t=-T(u)+R(t, u)$ for suitable perturbation terms $R(t, u)$ with $R(t, u) \rightarrow 0$ as $t \rightarrow+\infty$. The proof of convergence is based upon new a priori inequalities for solutions of such equations. (We remark that a discrete analogue of the ordinary method of steepest descent was applied by Vainberg in [15].)

2. We recall that a Banach space $X$ is said to satisfy condition $(\pi)_{C}$ for $C \geqq 1$, if $X$ is separable and if there exists a projectional system with constant $C$ in $X$, i.e. a sequence $\left\{X_{n}\right\}$ of finite dimensional subspaces of $X$, increasing with $n$ and with their union dense in $X$, and for each $n$, a projection $P_{n}$ of $X$ on $X_{n}$ (i.e. $P_{n}^{2}=P_{n}$ and $R\left(P_{n}\right)$ $=X_{n}$ ) for which $\left\|P_{n}\right\| \leqq C$. Every Banach space with a Schauder 
basis has a projectional system for some constant $C$, and a space with an absolute Schauder basis satisfies condition $(\pi)_{1}$.

Definition. Let $X$ be a Banach space with a projectional system, $T$ a mapping of $X$ into $X, f$ a given element of $X$. Then the equation $T(x)=f$ is said to be projectionally solvable in the strong (weak) sense if the following two assertions hold:

(a) For each $n \geqq 1$, the equation

$$
T_{n}\left(x_{n}\right)=P_{n} T\left(x_{n}\right)=P_{n}(f)
$$

has an unique solution $x_{n}$ in $X_{n}$, and exactly one.

(b) As $n \rightarrow+\infty$, these solutions $x_{n}$ converge strongly (weakly) in $X$ to $a$ solution $x$ of the equation $T(x)=f$.

Using the techniques of [8], Petryshyn [14] has shown that if a reflexive Banach space $X$ satisfies condition $(\pi)_{1}$ and has a weakly continuous duality mapping $J_{1}$ into $X^{*}$, then for every continuous mapping $T$ of $X$ into $X$ which maps bounded sets into bounded sets and satisfies an inequality of the form

$$
(T(x)-T(y), J(x-y)) \geqq \lambda(\|x-y\|)\|x-y\|
$$

for a function $\lambda$ from $R^{+}$to $R^{+}$which is continuous with $\lambda(0)=0$, $\lambda(r) \rightarrow+\infty$ as $r \rightarrow+\infty$, the equation $T(x)=f$ is projectionally solvable in the strong sense for each $f$ in $X$.

The arguments of Browder-de Figueiredo [8] and Petryshyn [14] do not apply without assumptions like that of the weak continuity of the mapping $J_{1}$. However, the writer has observed the following general fact which should be very useful in further investigations: If we know already that the equation $T(x)=f$ has a solution, then relatively weak assumptions ensure that the same equation is projectionally solvable in the strong sense.

THeOREM 7. Let $X$ be a separable Banach space with a projectional system $\left(\left\{X_{n}\right\},\left\{P_{n}\right\}\right)$ with constant $C \geqq 1, T$ a continuous mapping of $X$ into $X$, and let $T_{n}$ be the mapping of $X$ into $X$ given by $T_{n}=P_{n} T P_{n}$. Suppose that there exists a function $\lambda$ from $R^{+}$to $R^{+}$with $\lambda$ continuous and strictly increasing, $\lambda(0)=0$, such that for all $n$ :

$$
\left\|T_{n}(x)-T_{n}(y)\right\| \geqq \lambda(\|x-y\|), \quad\left(x, y \in X_{n}\right) .
$$

Let $f$ be a given element of $X$. Then the equation $T(x)=f$ has a solution $x$ in $X$ if and only if the same equation is projectionally solvable in the strong sense in $X$.

Proof of Theorem 7. Since projectional solvability implies solvability, it suffices to prove the converse. The inequality (6) implies 
that $T_{n}$ as a mapping of $X_{n}$ into $X_{n}$ is injective and has a closed range. Since $X_{n}$ is of finite dimension, the range is open by the Brouwer theorem of invariance of domain. Hence $R\left(T_{n}\right)=X_{n}$, and the equation $T_{n}\left(x_{n}\right)=P_{n} f$ has exactly one solution $x_{n}$ in $X_{n}$. It suffices to show that $x_{n} \rightarrow x$, where $x$ is a given solution of $T(x)=f$, whose existence we know by hypothesis. We know that

$$
T_{m} P_{n} x=P_{n} T x+P_{m}\left(T P_{n} x-T x\right)=P_{n} f+P_{n}\left(T P_{n} x-T x\right) .
$$

Since $T_{n}\left(x_{n}\right)=P_{n} f$ and both $x_{n}$ and $P_{n} f$ lie in $X_{n}$, we may apply the inequality (6) and obtain:

$$
\begin{aligned}
\lambda\left(\left\|P_{n} x-x_{n}\right\|\right) & \leqq\left\|T_{n} P_{n} x-T_{n} x_{n}\right\|=\left\|P_{n}\left(T P_{n} x-T x\right)\right\| \\
& \leqq C\left\|T P_{n} x-T x\right\| .
\end{aligned}
$$

However, given $\delta>0$, there exists $y_{n}$ in $X_{n}$ for $n$ sufficiently large such that $\left\|x-y_{n}\right\|<\delta$. For such $n$, we have $P_{n} x=P_{n} y_{n}+P_{n}\left(x-y_{n}\right)$ $=y_{n}+P_{n}\left(x-y_{n}\right)$, and therefore

$$
\left\|x-P_{n} x\right\| \leqq\left\|x-y_{n}\right\|+\left\|P_{n}\left(x-y_{n}\right)\right\| \leqq \delta+C \delta .
$$

It follows that $P_{n} x$ converges strongly to $x$ as $n \rightarrow \infty$. By the continuity of $T, T P_{n} x \rightarrow T x$. Thus:

$$
\begin{aligned}
\left\|x-x_{n}\right\| \leqq & \left\|x-P_{n} x\right\|+\left\|P_{n} x-x_{n}\right\| \leqq\left\|x-P_{n} x\right\| \\
& +\sigma\left(C\left\|T P_{n} x-T x\right\|\right)
\end{aligned}
$$

with $\sigma$ the inverse function of $\lambda$ which is continuous at zero. Hence $\left\|x-x_{n}\right\| \rightarrow 0$, and $x_{n}$ converges strongly to $x$ in $X$. q.e.d.

Remark. Petryshyn [14] has shown under the hypotheses of Theorem 7 that if $X$ if reflexive and $\lambda(r) \rightarrow+\infty$ as $r \rightarrow+\infty$, then the following condition is sufficient in order that the equation $T(x)=f$ be solvable in the strong sense for every $f$ in $X$ : (I) If $x_{n}$ converges weakly to $x$ with $x_{n} \in X_{n}$, and $T_{n} x_{n}$ converges strongly to $g$, then $T(x)=g$. For nonlinear accretive operators, however, a direct verification of condition (I) seems to demand a hypothesis of the existence of a weakly continuous duality mapping.

We now apply Theorem 7 to the projectional solvability of $T(x)=f$, with $T$ nonlinear and satisfying accretiveness conditions in a general Banach space.

THEOREM 8. Let $X$ be a Banach space with a strictly convex conjugate space $X^{*}$ and a duality mapping $J$ uniformly continuous from the unit sphere in $X$ to $X^{*}$. Suppose that $X$ has a projectional system 
( $\left.\left\{X_{n}\right\},\left\{P_{n}\right\}\right)$ with constant $C=1$. Let $T$ be a continuous mapping of $X$ into $X$, and suppose that there exists a continuous strictly increasing mapping $\lambda$ of $R^{+}$onto $R^{+}, \lambda(0)=0$, such that for all $x$ and $y$ of $X$,

$$
(T(x)-T(y), J(x-y)) \geqq \lambda(\|x-y\|)\|x-y\| .
$$

Then for every $f$ in $X$, the equation $T(x)=f$ is projectionally solvable in the strong sense for the projectional system $\left(\left\{X_{n}\right\},\left\{P_{n}\right\}\right)$.

Proof of TheOREM 8. It follows immediately from the inequality (7) that $T$ satisfies the conditions of Theorem 2, and therefore that the equation $T(x)=f$ has exactly one solution $x$ for each $f$ in $X$. We shall therefore derive the conclusion of projectional solvability from Theorem 7, and must verify the hypotheses of that theorem. It suffices to verify the inequality (6) with the same function $\lambda$ as in the hypothesis of Theorem 8 .

By the argument of [8], $P_{n}^{*} J w=J w$ for $w$ in $X_{n}$. Hence for $x$ and $y$ in $X_{n},\left(T_{n} x-T_{n} y, J(x-y)\right)=(T x-T y, J(x-y)) \geqq \lambda(\|x-y\|)\|x-y\|$, and

$$
\left\|T_{n} x-T_{n} y\right\| \geqq \lambda(\|x-y\|), \quad\left(x, y \in X_{n}\right) \text {. q.e.d. }
$$

\section{BIBLIOGRAPHY}

1. F. E. Browder, Existence and uniqueness theorems for solutions of nonlinear boundary value problems, Proc. Sympos. Appl. Math., Vol. 17, Amer. Math. Soc., Providence, R. I., 1965, pp. 24-29.

2. - Problèmes nonlinéaires, Univ. of Montreal Press, Montreal, 1966, $129 \mathrm{pp}$.

3. - - Fixed point theorems for nonlinear semicontractive mappings in Banach spaces, Arch. Rational Mech. Anal. 21 (1966), 259-269.

4. - Convergence of approximants to fixed points of nonexpansive mappings in Banach spaces, Arch Rational Mech. Anal, 24 (1967), 82-90.

5. - On the unification of the calculus of variations and the theory of monotone nonlinear operators in Banach spaces, Proc. Nat. Acad. Sci. U.S.A. 56 (1966), 419-425.

6. - Existence and approximation of solutions of nonlinear variational inequalities' Proc. Nat. Acad. Sci. U.S.A. 56 (1966), 1080-1086.

7. - Nonlinear equations of evolution and the method of steepest descent in Banach spaces, (to appear).

8. F. E. Browder and D. G. de Figueiredo, J-monotone nonlinear mappings in Banach spaces, Kon. Nederl. Akad. Wetesch. 69 (1966), 412-420.

9. P. Hartman, Generalized Lyapounov functions and nonlinear functional equations, Ann. Mat. Pura Appl. 69 (1965), 303-320.

10 G. Lumer and R. S. Phillips, Dissipative operators in Banach spaces, Pacific J. Math. 11 (1961), 679-698.

11. Ja. D. Mamedov, One-sided estimates in the conditions for asymptotic stability of solutions of differential equations with unbounded operators, Dokl. Akad. Nauk SSSR, 166 (1966), 533-535 = Soviet Math. Dokl. 7 (1966), 110. 
12. G. J. Minty, Monotone (nonlinear) operators in Hilbert space, Duke Math. J. 29 (1962), 341-346.

13. H. Murakami, On nonlinear ordinary and evolution equations, Univ. of Kansas Technical Report No. 9, June 1966.

14. W. V. Petryshyn, Projection methods in nonlinear numerical functional analysis, (to appear).

15. M. M. Vainberg, On the convergence of the process of steepest descent for nonlinear equations, Sibirsk. Mat. J. 2 (1961), 201-220.

\section{University of Chicago}

